\title{
Waste management system for petrochemical industries
}

\author{
Alelexei Bormotov ${ }^{1, *}$ and Ekaterina Kolobova ${ }^{1}$ \\ ${ }^{1}$ Penza State Technological University, 440039, Baydukov's passage, Gagarina street, building 1a/11, \\ Penza, Russia
}

\begin{abstract}
An oil refinery and the surrounding natural environment are presented as complex technical systems, as objects of research and modeling. The principles and methods of systems analysis, the theory of mathematical modeling and mathematical statistics, and correlation-regression analysis were used for this. Methodological principles of modeling the interaction of petrochemical production waste with the environment are proposed. On the basis of the proposed principles, a hierarchy of criteria and a systematic analysis of the system "petrochemical production - natural environment" are carried out. As the coordinates of modeling and control, it is proposed to take a set of indicators of the ecological state of an object in the vector space of ecological perfection, which sets all of its ecological, technical and economic properties in a single range of values. The system «petrochemical production - natural environment» is presented as a structural and logical model of a waste management system, showing the properties of synergy and emergence. Proposed methodological approaches and mathematical models of the system «petrochemical production - wastes from oil refineries - natural environment» allow to correctly assess technological risks, the effectiveness of the waste management system at petrochemical enterprises, the impact of unforeseen situations and accidents, the impact on the environment of waste from petrochemical enterprises, as well as the environmental and economic effect of the introduction of new technologies for oil refining and disposal of production waste.
\end{abstract}

\section{Introduction}

Oily waste (oil sludge) is formed at all stages of oil production and refining, and is the largest-tonnage petrochemical production waste of that has a significant negative impact on the natural environment (NE). Sulfur in oil is contained in the form of elemental sulfur, hydrogen sulfide, mercaptans, sulfides (theoethers) and disulfides (dithioesters), cyclic compounds and their homologues. A significant amount of sulfur is formed as a by-product of oil refining [1].

Since oil sludge (including reservoir type) contains in its composition an active organic part (oil products) capable of modifying most of the known binders, the mineral part, which can act as an effective filler to obtain composite materials with the required properties,

\footnotetext{
${ }^{*}$ Corresponding author: aleks21618@yandex.ru
} 
becomes possible to use tank-type oil sludge as a component for the manufacture of composites [2].

The first stage of the study of the system "petrochemical production (PP) - the natural environment (NE)", as well as waste management systems of petrochemical industries is the selection of those parts from the whole variety of components of these systems, the behavior of which will be studied in this work in as a subject modeling environment, namely, wastes from oil refineries (RW), refinery products (RP), technological parameters of production and the natural environment (NE), which affects humans and refineries, and which, in turn, is influenced by refineries and humans. The object of research is the PP-NE system, which is a complex technical system of interconnected elements, in which technological processes take place, which are to be studied using modeling.

\section{Research methods and principles}

The authors of the article used the principles and methods of systems analysis, the theory of mathematical modeling and mathematical statistics, correlation and regression analysis.

Modeling method. In the most general case, the system of PP-RW-NE from the side of the environment and the subject is influenced by a number of factors: $\bar{X}(t)-$ subject (human) exposure; $\bar{Z}(t)=\left[z_{1}(t), z_{2}(t), \ldots, z_{l}(t)\right]^{\mathrm{T}}$, one part $\overline{Z 1}(t)=$ $\left[z 1_{1}(t), z 1_{2}(t), \ldots, z 1_{l 1}(t)\right]^{\mathrm{T}}$ (controlled exposures) can be measured, another part $\bar{Z} 2(t)=\left[z 2_{1}(t), z 2_{2}(t), \ldots, z 2_{l 2}(t)\right]^{\mathrm{T}}$ (uncontrolled exposures) - is not measurable or not significant. The result of modeling at this stage is the establishment of the relationship between the output coordinates of the object $\bar{Y}(t)=\left[y_{1}(t), y_{2}(t), \ldots, y_{k}(t)\right]^{\mathrm{T}}$, characterizing the properties of PP products, control exposures $\bar{U}(t)=$ $\left[u_{1}(t), u_{2}(t), \ldots, u_{p}(t)\right]^{\mathrm{T}}$, coming from the subject through the technology of oil refining and fluctuations $\bar{Z}(t)[3-6]$.

\section{The main results}

As the coordinates of modeling and management, it is proposed to take a set of indicators of the ecological state of an object in the vector space of ecological perfection, which sets all its ecological, technical and economic properties in a single range of values.

Analysis of any component of the PP system as an object of environmental modeling, control and management assumes [7-9]:

- determination of the coordinates of the ecological state of the object - variables that characterize the behavior of an ecosystem element in the space of the vector of ecological states common for all components of the system;

- identification of controlled coordinates - output variables of an object, reflecting its ecological, technical and economic properties, subject to management in accordance with the tasks of the ecosystem;

- establishment of control actions - quantities, technical means, a set of actions of a technical and economic nature, through which the most rational management of the ecological state of the object;

- finding the disturbing influences - the input values of the control object, affecting the ecological state and controllable coordinates of the ecosystem, but the control of which is impractical at the present stage of development of science and technology or is not feasible;

- establishment of the internal parameters of the object of ecological research - values that determine its static and dynamic properties; 
- formation of environmental management criteria and restrictions on possible changes in the state of the object of environmental research under the influence of disturbances and changes in the internal parameters of the ecosystem.

The use of waste, oil products - technical sulfur (RW) and oil sludge tank tape (TTOL) - by combining them into an environmentally friendly composite material with a set of useful operational properties is the components of the vector of coordinates of the internal state (RW) and the vector of controlled coordinates (RP), Figure 1.

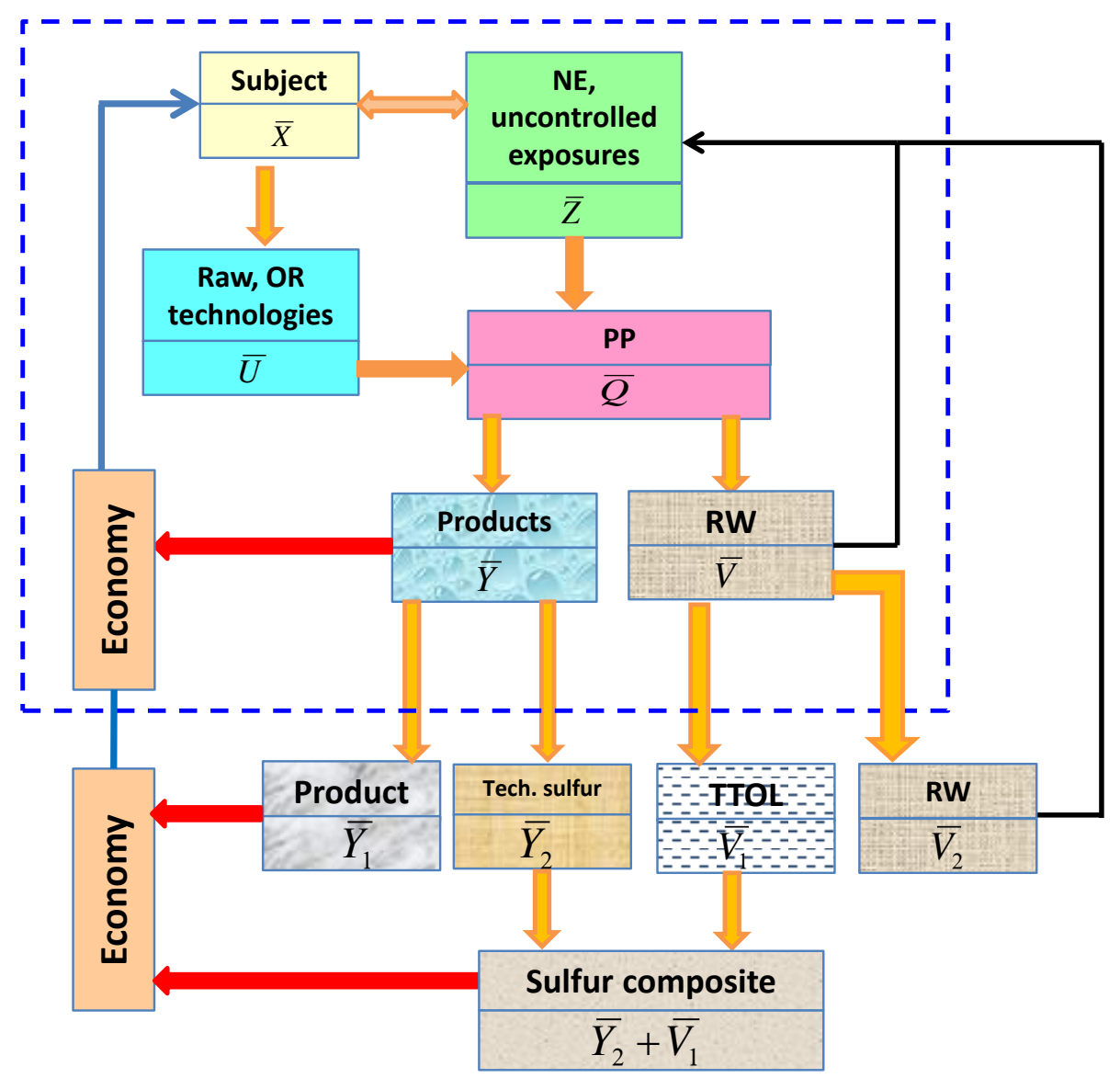

Fig. 1. Mathematical model of the system PP-RW-NE, NE - Natural Environment; PP petrochemical production; RW - refinery waste; TTOL - tank type oil sludge; OR - Oil refining

Before the introduction of a waste management system for petrochemical industries in the form of their joint utilization into an environmentally friendly composite material, the model of the PP-RW-NE system (in Figure 1 highlighted by a dotted line) according to formulas 3-5 can be represented as:

$$
\bar{Y}=f(\bar{X}(t), \bar{U}(t), \bar{Z}(t), \bar{Q})+\bar{V}(t)
$$

here vectors $\bar{X}(t) \bar{X}(t), \bar{U}(t), \bar{Z}(t)$ are variables relative to the vector $\bar{Y}$ via dynamic operator $\bar{\Theta}(t) ; \bar{Q}$ - system constraint vector; $\bar{V}(t)$ - vector of internal coordinates (constant value for the selected level of oil refining technologies), characterizing the environmental perfection of petrochemical production technologies and the amount of generated waste (RW). 
The use of waste, refined products - technical sulfur and TTOL - by combining them into an environmentally friendly composite material with a set of useful operational properties is the components of the vector of coordinates of the internal state (RW) and the vector of controlled coordinates (Products), Figure 1: $\bar{Y}=\bar{Y}_{1}+\bar{Y}_{2} ; \bar{V}=\bar{V}_{1}+\bar{V}_{2}$.

After the introduction of a waste management system for petrochemical industries in the form of their joint utilization into an environmentally friendly composite material, the model of the PP-RW-NE system is transformed into the following form:

$$
\bar{Y}_{1}+\left(\bar{Y}_{2}+\bar{V}_{1}\right)=f(\bar{X} ; \bar{Z} ; \bar{U})+\bar{V}_{2}
$$

here vectors $\bar{X}, \bar{U}, \bar{Z}$ are variables relative to the vector $\bar{Y} ; \bar{Q}$ - system constraint vector; $\bar{V}$ - vector of internal coordinates characterizing the amount of generated waste (NE), consisting of the sum of vectors: $\bar{V}_{2}$ - vector characterizing the amount of generated waste and $\bar{V}_{1}$ - vector characterizing the amount of tank type oil sludge from the total volume of RW; $\bar{Y}_{2}$ - vector characterizing the yield of technical sulfur as a by-product of oil refining; $\bar{Y}_{1}-$ vector characterizing the yield of oil refined products; $\left(\bar{Y}_{2}+\bar{V}_{1}\right)-$ the sum of vectors characterizing the yield of the sulfur composite.

As can be seen from the analysis, the structural-logical model of the waste management system before and after the introduction of the waste management system of petrochemical industries in the form of their joint disposal into an environmentally friendly composite material, the refinery product vector is: $R P=\bar{Y}_{1}+\bar{Y}_{2}+\bar{V}_{1}>\bar{Y}$, which indicates an increase in the output of a useful product into the economy, and the waste vector is: $R W=$ $\bar{V}-\overline{V_{1}}<\bar{V}$, which indicates a decrease in the environmental load on the fire protection system, an increase in the environmental safety of petrochemical production technologies and the effectiveness of the proposed solutions.

The structural-logical model of the waste management system presented in Figure 1 shows the properties of synergy and emergence - the appearance of the system's properties that are not inherent in the elements of the system; the fundamental irreducibility of the properties of the system to the sum of the properties of its constituent components (nonadditivity), i.e. the capabilities of the system exceed the sum of the capabilities of its constituent parts; overall system performance or functionality is better than a simple sum of elements. A new, previously absent, systemic effect appears - an environmentally friendly composite material with a set of useful performance properties. Thus, we have available all the characteristics and properties of the system, according to the existing concepts [10], which proves that the model presented in Figure 1 is a structural model of the waste management system.

\section{Discussion}

Modeling the PP-NE system is aimed at identifying its internal properties, structure and interrelationships of the elements that consist in the object of research, which are manifested in the interaction of PP and RW with the environment and the subject (human). There are many concepts of "object model". We will adopt an approach based on representing an object as a cybernetic system determined by a set of input actions

$$
\bar{Y}(t)=\{\bar{U}(t), \bar{Z}(t)\}=\left[x_{1}(t), x_{2}(t), \ldots, x_{r}(t)\right]^{T}
$$

here there are controlled $\bar{X}^{*}$ and uncontrolled $\bar{E}$, and many characteristics and limitations $\bar{Q}$, acting in the system and imposed on $\bar{X}^{*}$ and $\bar{E}, A=\left\{\bar{X}^{*}, \bar{E}, \bar{Q}\right\}$. 
The mathematical model of the cybernetic system establishes the mapping $\mathrm{F}$ of the given sets to the set of output coordinates of the object $\bar{Y}(t)=\left[y_{1}(t), y_{2}(t), \ldots, y_{k}(t)\right]^{\mathrm{T}}$ :

$$
F:=\left\{\bar{X}^{*}, \bar{E}, \bar{Q}\right\} \rightarrow \bar{Y}
$$

i.e.

$$
\bar{Y}=F\left(\bar{X}^{*}, \bar{E}, \bar{Q}\right)
$$

On a real object, a vector of input (control $\vec{U}=\left[u_{1}, u_{2} \ldots u_{n}\right]^{T}$ and vibration $\vec{Z}=$ $\left[z_{1}, z_{2} \ldots z_{n}\right]^{T}$ ) exposures $\vec{X}=\left[\begin{array}{llll}x_{1} & x_{2} \ldots x_{m}\end{array}\right]^{T}$, which is interpreted as a vector of a certain feature characterizing the influence of the operating conditions of the system under study, and a vector of output (controlled) coordinates $\vec{Y}=\left[\begin{array}{llll}y_{1} & y_{2} & \ldots & y_{n}\end{array}\right]^{T}$, interpreted as a vector of resultant indicators, response functions or performance indicators, characterizing the behavior or efficiency of functioning (quality) of the system.

The problem lies in the construction of a mathematical model of the system that establishes a cause-and-effect relationship between effective $\vec{Y}$ and definite $\vec{X}$ factors and constructive explanation of the behavior of the vector of functions $\vec{Y}$ effective attribute due to changes in factors - arguments $\vec{X}$, i.e. determining the class of feasible solutions $\mathrm{F}$ and in finding a vector function from this class that would give the best, in a certain sense, approximation of the behavior of the vector $\vec{Y}$ on a set of points - observations $\left\{x_{i}\right\} \mid i=$ $\overline{1, n}$.

Modeling the PP-NE technical system as a control object is aimed at defining the structure, i.e. identification of all existing input (disturbing, control) influences, state variables and output coordinates, as well as the relationship between them $[11,12]$.

PP and NE are multidimensional objects with controlled coordinates that characterize a set of operational properties, and therefore have several controlled and control variables, the aggregates of which in mathematical models are specified by vectors. The set of control and disturbing actions in the models is set by one vector of input actions.

Thus, both the technical and technological process or any element of the PP-NE system will be considered as a control object - this means to allocate the space in which the process to be controlled takes place, in space and time from the environment, that is, to determine it as a system of variables interconnected through the internal dynamic parameters of state - coordinates and controlled coordinates, control and disturbing influences.

We represent the model of the controlled object in the form of a set of quantities describing the functioning of the PP-RW-NE system and combining the following subsets (Figure 1) [13, 14].

Controlled coordinates (controlled variables) - a set of output values characterizing the behavior of the control object $\bar{Y}(t)$, the current values of which need to be controlled to achieve the assigned tasks. In the model, the controlled coordinates are set by the vector of controlled variables $\bar{Y}(t)=\left[y_{1}(t), y_{2}(t), \ldots, y_{k}(t)\right]^{T}$. In the physical sense, it is a complex of products of an oil refinery that has economic value.

The object state coordinates - are internal variables characterizing the state of petrochemical production technologies, the composition and structure of petrochemical production waste at certain points in time, i.e. the degree of environmental perfection of the technological process and the environmental load on the NE. In the model, the state coordinates are set by the state vector $\bar{V}(t)=\left[v_{1}(t), v_{2}(t), \ldots, v_{n}(t)\right]^{T}$, i.e. a sum of values of the state vector $\bar{V}\left(t_{1}\right), \bar{V}\left(t_{2}\right), \ldots, \bar{V}\left(t_{l}\right)$ at certain points in time $t_{1}, t_{2}, \ldots, t_{l}$. Controlled object coordinates $\bar{Y}(t)$ formed on the coordinates of its state $\bar{V}(t)$. The dimension of the 
vector of controlled coordinates $\mathrm{k}$ is less than or equal to the dimension of the state vector n. In a particular case, the controlled structure variables can coincide with the state coordinates. In the physical sense, these are the properties and structure of PP waste, assessed as "negative" for the environment.

Control exposures - a set of input values $\bar{U}(t) \in \bar{X}(t)$, by changing which we ensure the impact on the object in order to obtain the given parameters of products and the range of products of petrochemical industries. impact on the object in order to obtain the given parameters of products and the range of products of petrochemical industries. In the model, the control actions are set by the vector $\bar{U}(t)=\left[u_{1}(t), u_{2}(t), \ldots, u_{p}(t)\right]^{T}$. In the physical sense, these are the variable parameters of technologies for processing oil and related substances into products.

Vibrating exposures - set of uncontrolled input quantities $\bar{Z}(t) \in \bar{X}(t)$, whose changes have an undesirable effect on the state of the object $\bar{V}(t)$ and its controlled coordinates $\bar{Y}(t)$. In the model, the vibrating exposures are set by the vector $\bar{Z}(t)=$ $\left[z_{1}(t), z_{2}(t), \ldots, z_{l}(t)\right]^{T}$. The vector of vibrating exposures, in turn, can be divided into two components - the first can be measured, and the second cannot. The ability to measure the disturbing effect allows timely corrective changes in the technological process. In the physical sense, this is the effect of the environment and normal errors of technology: the inconstancy of the environment and its impact on production and humans (temperature, pressure, humidity, purity of water and air, concentration of harmful substances in water and air, etc.), normal errors in dosing and technological conversions, uneven distribution of physical and chemical properties of natural raw materials, impurities, human factor, etc.

Internal (own) dynamic parameters of the object - set of quantities $\bar{\theta}(t)$, characterizing the internal properties and quantitative cause-and-effect relationship of input and output variables in mathematical models of the object.

When considering the PP-RW-NE system, it is necessary from the internal parameters that characterize the properties of the products of petrochemical industries or the parameters of the technological process to go to the dynamic parameters that predetermine the internal properties of the object as an element of the control system in terms of the dynamics of its behavior. Such parameters, when presenting mathematical model in standard form, include static transfer coefficients $\mathrm{K}$, which determine the degree of transformation of an input variable of an element into an output variable in a static (steadystate) mode, and time constants $\mathrm{T}$, which determine the speed of the corresponding link or system [15].

\section{Conclusions}

Thus, the proposed method of mathematical modeling and analysis of the PP-RW-NE system as an object of modeling includes:

- determination of the coordinates of the object state - variables that characterize the behavior of the PP-RW-NE system in the state space;

- identification of controlled coordinates - output variables to be controlled in accordance with oil refining technology;

- establishment of control actions - values by means of which control in a given range of the output coordinates of the control object (PP products and waste from petrochemical industries) can be most effectively ensured;

- finding disturbing influences - input quantities of, affecting its controlled coordinates, but which cannot be changed with the help of a control device or which are not advisable to control (the action of the environment and technological redistributions); 
- identification of internal parameters of system - values that characterize the static and dynamic properties of an object (indicators of structures and characteristics of PP and NE);

- establishment of control criteria and restrictions on input and output variables, possible limits of change under the influence of disturbances in the internal parameters of the object (technological restrictions associated with the chosen technology of oil refining and waste disposal system).

The following stages of multi-criteria synthesis of the PP-RW-NE waste management system as a complex technical system based on the proposed mathematical model are proposed:

- formulation of management goals - synthesis of the PP-RW-NE system with the given structure and properties;

- definition of the control object - multilevel structures of the PP-RW-NE system and their parameters;

- creation of a control object model - nonlinear distributed dynamic models of various PP sublevels and linear kinetic models of the impact of oil refining waste on NE;

- control synthesis - creation of an algorithm for transition between structural levels with a solution to the problem of multifactor optimization;

- implementation of control - selection of components, selection of synthesis technology, obtaining the given parameters of the structure and properties of the PP-RWNE system.

The proposed methodological approaches and mathematical models of the PP-RW-NE system make it possible to correctly assess technological risks, the effectiveness of the waste management system at petrochemical enterprises, the impact of unforeseen situations and accidents, the impact on NE of waste from petrochemical enterprises, as well as the environmental and economic effect of introduction of new technologies for oil refining and waste disposal.

\section{References}

1. Le H.Tuan, S. Inozemtcev, E. Korolev, A. Grishina, The efficiency of sulfur modifier to neutralize toxic gases in sulfur-asphalt concrete technology, IOP Conference Series: Materials Science and Engineering 869 (3), 032016 (2020) doi:10.1088/1757899X/869/3/032016

2. M.A. Sharaf Eldean, A.M. Soliman, A novel study of using oil refinery plants waste gases for thermal desalination and electric power generation: Energy, exergy \& cost evaluations, Applied Energy 195, 453-477 https://doi.org/10.1016/j.apenergy.2017.03.052

3. E.A. Kolobova, Methodological principles of modelling oil refineries waste interaction with the natural environment, Oil Industry 5, 110-112 (2015)

4. Ch. Zhao, Yu. Li, Z. Gan, M. Nie, Method of smoldering combustion for refinery oil sludge treatment, Journal of Hazardous Materials 409, 124995 (2021) https://doi.org/10.1016/j.jhazmat.2020.124995

5. A. Bormotov, Development of quality functionals of kinetic processes for the synthesis of composite materials with specified parameters of structure and properties, E3S Web of Conferences 224, 02019 (2020) https://doi.org/10.1051/e3sconf/202022402019

6. M. Roudneshin, A. Azadeh, A novel multi-objective fuzzy model for optimiza-tion of oil sludge management by considering Health, Safety and Environment (HSE) and resiliency indicators in a gas refinery, Journal of Cleaner Production 206, 559-571 (2019) https://doi.org/10.1016/j.jclepro.2018.09.142 
7. V.M.F. Alexandre, T.M.S. de Castro, L.V. de Araújo, V.M.J. Santiago, D.M.G. Freire, M.C. Cammarota, Minimizing solid wastes in an activated sludge system treating oil refinery wastewater, Chemical Engineering and Processing: Process Intensification 103, 53-62 (2016) https://doi.org/10.1016/j.cep.2015.10.021

8. R.A Pandey, P.B Sanyal, N Chattopadhyay, S.N Kaul, Treatment and reuse of wastes of a vegetable oil refinery, Resources, Conservation and Recycling 37, (2), 101-117 (2003) https://doi.org/10.1016/S0921-3449(02)00071-X

9. Y. Sun, Y. Liu, J. Chen, Y. Huang, H. Lu, W. Yuan, Q. Yang, J. Hu, B. Yu, D. Wang, W. Xu, H. Wang, Physical pretreatment of petroleum refinery wastewater instead of chemicals addition for collaborative removal of oil and suspended solids, Journal of Cleaner Production 278, 123821 (2021) https://doi.org/10.1016/j.jclepro.2020.123821

10. T.H. Christensen, A. Damgaard, J. Levis, Y. Zhao, A. Björklund, U. Arena, M.A. Barlaz, V. Starostina, A. Boldrin, T.F. Astrup, V. Bisinella. Application of LCA modelling in integrated waste management, Waste Management 118, 313-322 (2020) https://doi.org/10.1016/j.wasman.2020.08.034

11. X. Zhen, W. Cheng, Ng. Fendy, Y.W. Tong, Y. Dai, K.G. Neoh, C-H. Wang, Toxicity assessment of carbon black waste: A by-product from oil refineries, Journal of $\begin{array}{llll}\text { Hazardous } & \text { Materials } & 321, & 600-610\end{array}$ https://doi.org/10.1016/j.jhazmat.2016.09.043

12. A. Tsiligiannis, Ch. Tsiliyannis. Oil refinery sludge and renewable fuel blends as energy sources for the cement industry, Renewable Energy 157, 55-70 (2020) https://doi.org/10.1016/j.renene.2020.03.129

13. Yu.M. Bazhenov, A.P. Proshin, A.I. Eremkin, E.V. Korolev, A.N. Bormotov, Superheavy concrete for protection against radiation, Construction Materials 8, 6-9 (2005)

14. A.P. Proshin, E.V. Korolev, A.N. Bormotov, O.L. Figovsky, The extra-heavy concrete for protection from radiation, Proceedings of the International Conference on Role of Concrete in Nuclear Facilities 69-76 (2005)

15. T.K. Akchurin, V.D. Tukhareli, O.Yu. Pushkarskaya, The Modifying Additive for Concrete Compositions Based on the Oil Refinery Waste, Procedia Engineering 150, 1485-1490 (2016) https://doi.org/10.1016/j.proeng.2016.07.087 